\title{
Electron-cyclotron absorption in high-temperature plasmas: quasi-exact analytical evaluation and comparative numerical analysis $\ddagger$
}

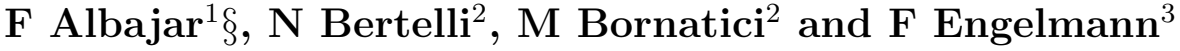 \\ ${ }^{1}$ Departament de Física i Enginyeria Nuclear, Universitat Politècnica de Catalunya, \\ 08028 Barcelona, Spain \\ ${ }^{2}$ Physics Department "A. Volta", University of Pavia, I-27100 Pavia, Italy \\ ${ }^{3}$ Max-Planck-Institut für Plasmaphysik, 85748 Garching, Germany \\ E-mail: bornatici@fisicavolta.unipv.it
}

\begin{abstract}
On the basis of the electromagnetic energy balance equation, a quasi-exact analytical evaluation of the electron cyclotron (EC) absorption coefficient is performed for arbitrary propagation (with respect to the magnetic field) in a (Maxwellian) magneto-plasma for the temperature range of interest for fusion reactors (in which EC radiation losses tend to be important in the plasma power balance). The calculation makes use of Bateman's expansion for the product of two Bessel functions, retaining the lowest order contribution. The integration over electron momentum can then be carried out analytically, fully accounting for finite Larmor radius effects in this approximation. On the basis of the analytical expressions for the EC absorption coefficients of both the extraordinary and ordinary mode thus obtained, (i) for the case of perpendicular propagation simple formulae are derived for both modes and (ii) a numerical analysis of the angular distribution of EC absorption is carried out. An assessment of the accuracy of asymptotic expressions that have been given earlier is also performed, showing that these approximations can be usefully applied for calculating EC power losses from reactor-grade plasmas.
\end{abstract}

\section{Introduction}

It has been recognised that in the magneto-plasma regimes as anticipated for fusion reactor operation electron cyclotron (EC) losses may play an important part in the power balance of the hot plasma core (see, e.g., Albajar et al 2002). Therefore, an accurate and fast procedure for solving the equation of radiative transfer for EC waves is needed that can be used in the context of power (and particle) transport calculations. In this paper, a quasi-exact analytical formula for the absorption coefficient of EC waves valid for mildly relativistic Maxwellian magneto-plasmas is deduced which allows simplifying the

$\ddagger$ Presented in part at the 14th Joint Workshop on Electron Cyclotron Emission and Electron Cyclotron Resonance Heating, Santorini, Greece, May 9-12, 2006.

$\S$ Present address: EFDA, Max-Planck-Institut für Plasmaphysik, 85748 Garching, Germany. 
calculation of the absorption coefficient and also can be used to determine the accuracy of still simpler asymptotic expressions as available in literature.

As in the regimes considered the wave damping is weak, the (collisionless) absorption coefficient $\alpha \equiv 2 \operatorname{Im} \mathbf{k} \cdot \mathbf{S} /|\mathbf{S}|$ of a wave having a (real) frequency $\omega$ and a (complex) wave vector $\mathbf{k}$, that is the spatial damping rate of the electromagnetic energy along the direction of the energy flux $\mathbf{S}(\mathbf{k}, \omega)$, is most conveniently evaluated starting from the electromagnetic energy balance with the result that (Bornatici et al 1983, Bornatici and Engelmann 1994)

$$
\alpha=\frac{\omega}{c}\left(\mathbf{e}^{*} \cdot \varepsilon_{a} \cdot \mathbf{e}\right)
$$

where $\mathbf{e} \equiv \mathbf{E}(\mathbf{k}, \omega) /(4 \pi|\mathbf{S}(\mathbf{k}, \omega)| / c)^{1 / 2}$ is the normalized wave electric field, $\boldsymbol{\varepsilon}_{a} \equiv \boldsymbol{\varepsilon}_{a}(\mathbf{k}, \omega)$ is the anti-Hermitian part of the (collisionless) dielectric tensor, and $c$ is the speed of light. On making use of the dielectric tensor expressed as an expansion in electron cyclotron harmonics, for a magneto-plasma the EC absorption coefficient (1) takes the form (Bornatici and Engelmann 1994)

$$
\alpha=-\pi \frac{\omega_{p}^{2}}{c \omega} \sum_{n=1}^{\infty} \int \frac{d^{3} u}{\gamma} \delta\left(\gamma-N_{\|} u_{\|}-\frac{n}{\bar{\omega}}\right)\left|\mathbf{e} \cdot \mathbf{V}_{n}^{*}\right|^{2} \hat{R}_{n} f,
$$

the $\delta$ function accounting for the relativistic EC resonance condition

$$
\gamma-N_{\|} u_{\|}-\frac{n}{\bar{\omega}}=0
$$

Furthermore, adopting Cartesian coordinates such that wave propagation is in the $x, z$ plane,

$$
\begin{aligned}
\mathbf{V}_{n} & \equiv \frac{n}{\bar{\omega} N_{\perp}}\left(J_{n}, \frac{i b}{n} J_{n}^{\prime}, \frac{\bar{\omega} N_{\perp}}{n} u_{\|} J_{n}\right) \\
\hat{R}_{n} & \equiv \frac{n}{\bar{\omega}} \frac{1}{u_{\perp}} \frac{\partial}{\partial u_{\perp}}+N_{\|} \frac{\partial}{\partial u_{\|}}
\end{aligned}
$$

with $J_{n} \equiv J_{n}(b)$ the Bessel function of the first kind of (integer) order $n(n \geq 1$ is the harmonic number) and argument $b \equiv \bar{\omega} N_{\perp} u_{\perp}$, and $J_{n}^{\prime} \equiv d J_{n} / d b$. In (2), $\omega_{p}$ is the electron plasma frequency and $\bar{\omega} \equiv \omega / \omega_{c}$ with $\omega_{c}=e B_{0} / m c(>0)$ the nonrelativistic EC frequency; $\gamma \equiv\left(1+u^{2}\right)^{1 / 2}$ with $\mathbf{u} \equiv \mathbf{p} / m c$ the (dimensionless) electron momentum; $\mathbf{N} \equiv \mathbf{k} c / \omega$ is the refractive index with the subscript $\|(\perp)$ denoting the component parallel (perpendicular) to the equilibrium magnetic field $\mathbf{B}_{0}\left(=B_{0} \hat{\mathbf{z}}\right) ; f \equiv f\left(u_{\perp}, u_{\|}\right)$is the (gyrotropic) electron distribution function (normalized to unity). In particular, for an isotropic distribution $f=f(\gamma)$, one has, cf. $(2 a), \hat{R}_{n} f=d f / d \gamma$ upon using $(2 d)$ along with the resonance condition $(2 b)$.

With reference to $(2 a)$, the factor

$$
\left|\mathbf{e} \cdot \mathbf{V}_{n}^{*}\right|^{2}=\left(\frac{n}{\bar{\omega} N_{\perp}}\right)^{2}\left|\left(e_{x}+\frac{\bar{\omega} N_{\perp}}{n} u_{\|} e_{z}\right) J_{n}(b)-\frac{i b}{n} J_{n}^{\prime}(b) e_{y}\right|^{2}
$$

as follows from using $(2 c)$, accounts for the effects of both the wave polarization and the finiteness of the (electron) Larmor radius, the latter being weighed by the argument 
$b$ of the Bessel functions. For the evaluation of (3), one usually proceeds to a series expansion of the Bessel functions in powers of $b$, retaining only the lowest significant order terms (Bornatici et al 1983, Bornatici and Engelmann 1994). Here, instead, we adopt a different procedure based on Bateman's expansion for the product of two Bessel functions (Watson 1944, Granata 1990), such an approach having been employed to evaluate the EC absorption in the context of a (numerical) solution of the dispersion relation (Granata and Fidone 1991, Albajar 2001). In this way a quasi-exact (QE) analytical formula for the EC absorption coefficient of a plasma in thermodynamic equilibrium can be deduced that remains applicable for mildly relativistic plasma temperatures, except for very low frequencies $(\bar{\omega} \lesssim 2)$ which, however, are not important for the evaluation of the EC power loss of such plasmas, as here most of the EC radiative power tends to be concentrated in higher harmonics.

The derivation of the QE analytical formula for the EC absorption coefficient is given in Section 2. In Section 3, for reference the asymptotic expressions for the EC absorption coefficient for $\bar{\omega} \gg 1$ (in which case strong overlap of different harmonic contributions is present in a mildly relativistic plasma) derived by Robinson (1985) and by Trubnikov (1979) are recalled for the particularly important case of wave propagation perpendicular to the magnetic field. A numerical analysis of the analytical result derived in Section 2 is performed in Section 4, along with an assessment of the accuracy of the asymptotic expressions for the EC absorption coefficient obtained by Robinson and Trubnikov. The conclusions are summarized in Section 5. The necessary mathematical apparatus and details of calculation are described in Appendixes A and B.

\section{Quasi-exact analytical evaluation of the EC absorption coefficient}

With reference to the $\mathbf{u}$-integration in $(2 a)$, let us adopt cylindrical coordinates, for which $d^{3} u=2 \pi d u_{\|} u_{\perp} d u_{\perp}$, and express the $\delta$ function in a form that makes the integration over the $u_{\perp}$-variable straightforward, i.e.,

$$
\delta\left(\gamma-N_{\|} u_{\|}-\frac{n}{\bar{\omega}}\right)=\frac{\gamma}{u_{\perp}} \delta\left(u_{\perp}-u_{\perp}\left(u_{\|}\right)\right)
$$

where

$u_{\perp}\left(u_{\|}\right) \equiv\left[\left(1-N_{\|}^{2}\right)\left(u_{+}-u_{\|}\right)\left(u_{\|}-u_{-}\right)\right]^{1 / 2}, \quad N_{\|}^{2}<1, \quad u_{-} \leq u_{\|} \leq u_{+}$,

with

$u_{ \pm} \equiv \frac{1}{\sqrt{1-N_{\|}^{2}}}\left\{\frac{n}{n_{0}} N_{\|} \pm\left[\left(\frac{n}{n_{0}}\right)^{2}-1\right]^{1 / 2}\right\}, \quad n \geq n_{0} \equiv \bar{\omega} \sqrt{1-N_{\|}^{2}}$

$\left(N_{\|}>0\right.$ is considered). As for the $u_{\|}$-integration, let us make the change of variable

$$
u_{\|}=\frac{1}{\sqrt{1-N_{\|}^{2}}}\left\{\frac{n}{n_{0}} N_{\|}+\left[\left(\frac{n}{n_{0}}\right)^{2}-1\right]^{1 / 2} t\right\} \equiv u_{\|}(t)
$$


such that the range of integration $u_{-} \leq u_{\|} \leq u_{+}$, cf. (4b), is mapped onto $-1 \leq t \leq 1$. With (5) into (4b), one gets

$$
u_{\perp}\left(u_{\|}\right)=\left[\left(\frac{n}{n_{0}}\right)^{2}-1\right]^{1 / 2} \sqrt{1-t^{2}} \equiv u_{\perp}\left(t^{2}\right)
$$

the quantity $b \equiv \bar{\omega} N_{\perp} u_{\perp}$ taking the form

$$
b=x_{n}\left(N_{\perp}\right) \sqrt{1-t^{2}} \equiv b\left(t^{2}\right) \quad \text { with } \quad x_{n}\left(N_{\perp}\right) \equiv \bar{\omega} N_{\perp}\left[\left(\frac{n}{n_{0}}\right)^{2}-1\right]^{1 / 2}
$$

As a result, one gets the absorption coefficient $(2 a)$, normalized to $\omega_{p}^{2} / c \omega_{c}$, i.e., $\alpha /\left(\omega_{p}^{2} / c \omega_{c}\right) \equiv \bar{\alpha}$, in the form

$$
\bar{\alpha}=-\frac{2 \pi^{2}}{n_{0}} \sum_{n \geq n_{0}}\left[\left(\frac{n}{n_{0}}\right)^{2}-1\right]^{1 / 2} \int_{-1}^{1} d t\left|\mathbf{e} \cdot \mathbf{V}_{n}^{*}\right|^{2} \hat{R}_{n} f
$$

the integrand being a function of $t$ through $u_{\perp}=u_{\perp}\left(t^{2}\right), b=b\left(t^{2}\right)$ and $u_{\|}=u_{\|}(t)$, as given, respectively, by $(6 a),(6 b)$ and (5).

As for the "polarization factor" $\left|\mathbf{e} \cdot \mathbf{V}_{n}^{*}\right|^{2}$, cf. (3), along with (5) and (6b), one has

$$
\begin{aligned}
\left|\mathbf{e} \cdot \mathbf{V}_{n}^{*}\right|^{2} & =\left(\frac{n}{\bar{\omega} N_{\perp}}\right)^{2}\left\{\left[\left|A_{x z}\right|^{2}+\left|e_{y}\right|^{2}+\operatorname{Re}\left(i A_{x z} e_{y}^{*}\right) \frac{x_{n}}{n} \frac{\partial}{\partial x_{n}}\right] J_{n}^{2}+\right. \\
& -\left(\frac{x_{n} \sqrt{1-t^{2}}}{n}\right)^{2}\left|e_{y}\right|^{2} J_{n-1} J_{n+1}+\left(\frac{x_{n}}{n \sqrt{1-N_{\|}^{2}}}\right)^{2}\left|e_{z}\right|^{2}\left(t J_{n}\right)^{2}+ \\
& \left.+\frac{x_{n}}{n \sqrt{1-N_{\|}^{2}}}\left[2 \operatorname{Re}\left(A_{x z} e_{z}^{*}\right)+\operatorname{Re}\left(i e_{y}^{*} e_{z}\right) \frac{x_{n}}{n} \frac{\partial}{\partial x_{n}}\right]\left(t J_{n}^{2}\right)\right\}
\end{aligned}
$$

where

$$
A_{x z} \equiv e_{x}+\frac{N_{\perp} N_{\|}}{1-N_{\|}^{2}} e_{z}
$$

the argument of the Bessel functions in $(8 a)$ being $\left(x_{n} \sqrt{1-t^{2}}\right)$, cf. (6b). To obtain expression ( $8 a$ ) use has been made of the relation

$$
\left[x J_{n}^{\prime}(x)\right]^{2}=\left[n J_{n}(x)\right]^{2}-x^{2} J_{n-1}(x) J_{n+1}(x) .
$$

Regarding the integration in (7), it is worth noting that the last term of $(8 a)$, proportional to $t\left[J_{n}\left(x_{n} \sqrt{1-t^{2}}\right)\right]^{2}$, is odd in $t$, the rest of $(8 a)$, instead, being even in $t$.

For reference later on, let us consider explicitly the case of propagation perpendicular to the magnetic field $\mathbf{B}_{0}=B_{0} \hat{\mathbf{z}}$, that is, propagation along the $\hat{\mathbf{x}}$-direction. In this case, the two fundamental propagating modes are decoupled in the coordinates used, the extraordinary $(X)$ mode being elliptically polarized in the $(x, y)$-plane, i.e., $e_{z}=0$, and 
the ordinary $(O)$ mode being linearly polarized along the $\hat{\mathbf{z}}$-direction, i.e., $e_{x}=e_{y}=0$. Thus, the polarization factor (8) takes the form

$$
\left|\mathbf{e} \cdot \mathbf{V}_{n}^{*}\right|^{2}\left(N_{\|}=0\right)=\left\{\begin{array}{l}
\left(\frac{n}{\bar{\omega} N_{\perp}}\right)^{2}\left\{\left[\left|e_{x}\right|^{2}+\left|e_{y}\right|^{2}+R e\left(i e_{x} e_{y}^{*}\right) \frac{x_{n}}{n} \frac{\partial}{\partial x_{n}}\right] J_{n}^{2}+\right. \\
\left.-\left(\frac{x_{n} \sqrt{1-t^{2}}}{n}\right)^{2}\left|e_{y}\right|^{2} J_{n-1} J_{n+1}\right\}, \quad \text { for the X-mode, } \\
{\left[\left(\frac{n}{\bar{\omega}}\right)^{2}-1\right]\left(t J_{n}\right)^{2}\left|e_{z}\right|^{2}, \quad \text { for the O-mode }}
\end{array}\right.
$$

with the harmonic number $n \geq \bar{\omega}$. Note that the polarization factor for perpendicular propagation according to (9) is an even function of $t$.

To carry out the $t$-integration in (7) explicitly, one needs to specify the distribution function $f$, which now is assumed to be a relativistic isotropic Maxwellian, for which, cf. $(2 d)$,

$$
\begin{aligned}
& \hat{R}_{n} f=\frac{d f}{d \gamma}=-\mu f=-\frac{a(\mu)}{(2 \pi)^{3 / 2}} \mu^{5 / 2} e^{-\mu(\gamma-1)}, \\
& \mu \equiv \frac{m c^{2}}{T_{e}}, \quad a(\mu) \equiv\left(\frac{\pi}{2 \mu}\right)^{1 / 2} e^{-\mu} / K_{2}(\mu),
\end{aligned}
$$

$T_{e}$ being the electron temperature and $K_{2}(\mu)$ being the Macdonald function. Along with (10), the EC absorption coefficient (7) is

$$
\bar{\alpha}\left(\bar{\omega}, N_{\|}, N_{\perp}\right)=\sqrt{\frac{\pi}{2}} \frac{a(\mu) \mu^{5 / 2}}{n_{0}} \sum_{n \geq n_{0}}\left[\left(\frac{n}{n_{0}}\right)^{2}-1\right]^{1 / 2} P_{n}\left(\bar{\omega}, N_{\|}, N_{\perp}\right) e^{-\mu\left(\frac{n / n_{0}}{\sqrt{1-N_{\|}^{2}}}-1\right)}
$$

where

$$
P_{n}\left(\bar{\omega}, N_{\|}, N_{\perp}\right) \equiv \int_{-1}^{1} d t\left|\mathbf{e} \cdot \mathbf{V}_{n}^{*}\right|^{2} e^{-y_{n}\left(N_{\|}\right) t}
$$

with

$$
y_{n}\left(N_{\|}\right) \equiv \frac{\mu N_{\|}}{\sqrt{1-N_{\|}^{2}}}\left[\left(\frac{n}{n_{0}}\right)^{2}-1\right]^{1 / 2} .
$$

The integral (11b) along with (8a) can be evaluated analytically with high accuracy on making use of Bateman's expansion for the product of two Bessel functions, cf. Appendix A, retaining just low order contributions. One thus obtains (the details of the evaluation of (11b) are given in Appendix B) for propagation at large enough angles 
with respect to the magnetic field,

$$
\begin{aligned}
P_{n} & =\pi g_{n}\left(\frac{n}{\bar{\omega} N_{\perp}}\right)^{2}\left\{\left[\left|A_{x z}\right|^{2}+\left|e_{y}\right|^{2}+\operatorname{Re}\left(i A_{x z} e_{y}^{*}\right) \frac{x_{n}}{n} \frac{\partial}{\partial x_{n}}+\right.\right. \\
& -\left(\frac{x_{n}}{n}\right)^{2} \frac{n}{n+1}\left|e_{y}\right|^{2}\left(1-\frac{\partial^{2}}{\partial y_{n}^{2}}\right)+\left(\frac{x_{n}}{n \sqrt{1-N_{\|}^{2}}}\right)\left|e_{z}\right|^{2} \frac{\partial^{2}}{\partial y_{n}^{2}}+ \\
& \left.-\frac{x_{n}}{n \sqrt{1-N_{\|}^{2}}}\left(2 \operatorname{Re}\left(A_{x z} e_{z}^{*}\right)+\operatorname{Re}\left(i e_{y}^{*} e_{z}\right) \frac{x_{n}}{n} \frac{\partial}{\partial x_{n}}\right) \frac{\partial}{\partial y_{n}}\right] \frac{\left|J_{n+1 / 2}\left(z_{n}\right)\right|^{2}}{x_{n}}+ \\
& \left.+\left(\frac{x_{n}}{n}\right)^{2} \frac{2 n+3}{(n+1)(n+2)}\left|e_{y}\right|^{2}\left(1-\frac{\partial^{2}}{\partial y_{n}^{2}}\right) \frac{\left|J_{n+3 / 2}\left(z_{n}\right)\right|^{2}}{x_{n}}\right\},
\end{aligned}
$$

with $g_{n} \equiv(2 n+1) ! /\left(2^{n} n !\right)^{2}, A_{x z}, x_{n}\left(\equiv x_{n}\left(N_{\perp}\right)\right)$ and $y_{n}\left(\equiv y_{n}\left(N_{\|}\right)\right)$being defined, respectively, by (8b), (6b) and (11c); the (complex) argument of the Bessel functions $J_{n+1 / 2}$ and $J_{n+3 / 2}$ is

$$
z_{n}\left(\equiv z_{n}\left(x_{n}\left(N_{\perp}\right), y_{n}\left(N_{\|}\right)\right)\right) \equiv \frac{1}{2}\left(\sqrt{4 x_{n}^{2}-y_{n}^{2}}+i y_{n}\right)
$$

the angular range for which $(12 a)$ is useful in practice being

$$
4 x_{n}^{2}-y_{n}^{2}>0 \text {, i.e., } 2 n_{0}>\mu N_{\|} / N_{\perp} \text {. }
$$

In particular, for perpendicular propagation, for which $y_{n}=0$, cf. $(11 c), z_{n}=x_{n}=$ $N_{\perp} \sqrt{n^{2}-\bar{\omega}^{2}}$ from (6b). For propagation at angles for which condition (12c) is reversed, i.e., propagation at smaller angles to the magnetic field, it is convenient to replace both the Bessel functions of the first kind occurring in (12a) by modified Bessel functions of the first kind, of the same order and real arguments, i.e.,

$$
\left|J_{n+1 / 2}\left(z_{n}\right)\right|^{2} \rightarrow I_{n+1 / 2}\left(z_{n}^{+}\right) I_{n+1 / 2}\left(z_{n}^{-}\right) \text {and }\left|J_{n+3 / 2}\left(z_{n}\right)\right|^{2} \rightarrow I_{n+3 / 2}\left(z_{n}^{+}\right) I_{n+3 / 2}\left(z_{n}^{-}\right),
$$

the (real) arguments $z_{n}^{ \pm}$being

$$
z_{n}^{ \pm} \equiv \frac{1}{2}\left(y_{n} \pm \sqrt{y_{n}^{2}-4 x_{n}^{2}}\right)
$$

with

$$
y_{n}^{2}-4 x_{n}^{2}>0 \text {, i.e., } 2 n_{0}<\mu N_{\|} / N_{\perp} .
$$

The expressions (11)-(12), to be referred to as quasi-exact analytical formula for the EC absorption coefficient, are the main result of this paper.

The mode polarization, entering into $(12 a)$ via the normalized electric field vector $\mathbf{e}$, is obtained from the wave equation in terms of the Hermitian part of the dielectric tensor, which can be taken in the cold-plasma limit. As is well-known (Bornatici et $a l, 1983)$, the cold-plasma approximation is adequate for the harmonics $n \geq 3$, in the practically relevant case $\omega^{2}\left(\simeq\left(n \omega_{c}\right)^{2}\right)>\omega_{p}^{2}$, as well as for the second harmonic provided that $\omega_{p}^{2} \ll \omega_{c}^{2}$. Instead, the case of the first harmonic, as well as the case of the second harmonic for $\omega_{p}^{2} \gtrsim \omega_{c}^{2}$, must be dealt with differently from the higher $(n \geq 3)$ harmonics. However, being concerned with the evaluation of the EC losses from hot plasmas, for 
which the main contribution to the sum over harmonics in (11a) stems from the higher harmonics, there is no need to treat the low harmonics, and, in particular, the first one, accurately. In the cold-plasma limit, one has, cf. Eq.(3.1.61), p. 1198, of Bornatici et al (1983),

$$
e_{x}=i \bar{\omega}\left[1-\left(1-\frac{1}{\bar{\omega}^{2}}\right) f^{(i)}(\vartheta)\right] e_{y}
$$

where, cf. Tab.VIII, p. 1196, of Bornatici et al (1983),

$$
f^{(i)}(\vartheta) \equiv \frac{2\left[1-\left(\omega_{p} / \omega\right)^{2}\right]}{2\left[1-\left(\omega_{p} / \omega\right)^{2}\right]-\frac{\sin ^{2} \vartheta \mp \rho(\vartheta)}{\bar{\omega}^{2}}}
$$

with

$$
\rho^{2}(\vartheta) \equiv \sin ^{4} \vartheta+4 \bar{\omega}^{2}\left[1-\left(\frac{\omega_{p}}{\omega}\right)^{2}\right]^{2} \cos ^{2} \vartheta, \quad \vartheta \equiv \varangle\left(\mathbf{k}, \mathbf{B}_{0}\right),
$$

the minus and plus sign in (13b) referring, respectively, to the $O$-mode $(i=O)$ and the $X$-mode $(i=X)$. Furthermore, cf. Eq.(3.1.62), p. 1198, of Bornatici et al (1983),

$$
e_{z}=-\frac{\left[N^{(i)}\right]^{2} \sin \vartheta \cos \vartheta}{1-\left(\frac{\omega_{p}}{\omega}\right)^{2}-\left[N^{(i)}\right]^{2} \sin ^{2} \vartheta} e_{x}
$$

with, cf. Tab.VIII, p. 1196, of Bornatici et al (1983),

$$
\left[N^{(i)}\right]^{2}=1-\left(\frac{\omega_{p}}{\omega}\right)^{2} f^{(i)}(\vartheta)
$$

the (cold) dispersion relation of the mode $i$.

On making use of (13), the effect of the "polarization" vector $\mathbf{e}$ in (12a) can be expressed in terms of $\left|e_{y}\right|^{2}\left(\equiv\left|E_{y}\right|^{2} /\left(\frac{4 \pi}{c}|\mathbf{S}|\right)\right)$. In the cold-plasma limit, the electromagnetic energy flux $\mathbf{S}$ is well approximated by the Poynting vector

$$
\mathbf{P}=[a(\vartheta) \hat{\mathbf{x}}+b(\vartheta) \hat{\mathbf{z}}] N c \frac{\left|E_{y}\right|^{2}}{4 \pi}
$$

so that

$$
\left|e_{y}\right|^{2}=\frac{1}{N\left[a^{2}(\vartheta)+b^{2}(\vartheta)\right]^{1 / 2}}, \quad N \equiv N^{(i)},
$$

with $N=N^{(i)}$ given by (13e), $a(\vartheta)$ and $b(\vartheta)$ being given, respectively, by Eqs.(3.1.78a) and (3.1.78b), p. 1204, of Bornatici et al (1983), with $n \rightarrow \bar{\omega}$.

For the case of propagation perpendicular to the magnetic field, i.e., along the $\hat{\mathbf{x}}$-direction, for the $X$-mode for which $e_{z}=0,(12 a)$ reduces to

$$
\begin{aligned}
P_{n}^{(X)}\left(N_{\|}\right. & =0)=\pi g_{n}\left(\frac{n}{\bar{\omega} N_{\perp}^{(X)}}\right)^{2}\left\{\left[\left|e_{x}\right|^{2}+\left|e_{y}\right|^{2}+R e\left(i e_{x} e_{y}^{*}\right) \frac{x_{n}}{n} \frac{\partial}{\partial x_{n}}+\right.\right. \\
& \left.-\left(\frac{x_{n}}{n}\right)^{2} \frac{n}{n+1}\left|e_{y}\right|^{2}\right] \frac{J_{n+1 / 2}^{2}\left(x_{n}\right)}{x_{n}}+\frac{x_{n}}{n^{2}} \frac{1}{(n+1)}\left|e_{y}\right|^{2}\left[\frac{2 n+3}{n+2} J_{n+3 / 2}^{2}\left(x_{n}\right)+\right. \\
& \left.\left.-n \frac{\partial^{2}\left|J_{n+1 / 2}\left(z_{n}\right)\right|^{2}}{\partial y_{n}^{2}}-\frac{2 n+3}{n+2} \frac{\partial^{2}\left|J_{n+3 / 2}\left(z_{n}\right)\right|^{2}}{\partial y_{n}^{2}}\right]\right\}
\end{aligned}
$$


the second derivatives with respect to $y_{n}$, being evaluated for $y_{n}=0$, are given by (B.12) and (B.13) of Appendix B. Furthermore (13a) and (14b) yield, for $\vartheta=\pi / 2$,

$e_{x}=-i \frac{\omega_{p}^{2}}{\bar{\omega} \omega^{2}}\left[1-\left(\frac{\omega_{p}}{\omega}\right)^{2}-\frac{1}{\bar{\omega}^{2}}\right]^{-1} e_{y} \equiv-i A_{\perp} e_{y}, \quad\left|e_{y}\right|^{2}=\frac{1}{N_{\perp}^{(X)}}$,

and from $(15 a)$ one obtains

$$
\begin{aligned}
P_{n}^{(X)}\left(N_{\|}=0\right) & =\frac{\pi g_{n}}{\left(N_{\perp}^{(X)}\right)^{3} x_{n}}\left\{\left(1+A_{\perp}\right)^{2}\left(\frac{n}{\bar{\omega}} J_{n+1 / 2}\right)^{2}-\frac{x_{n}}{\bar{\omega}}\left[2 A_{\perp} \frac{n}{\bar{\omega}} J_{n+1 / 2} J_{n+3 / 2}+\right.\right. \\
& \left.\left.+\frac{1}{2(n+1)} \frac{x_{n}}{\bar{\omega}} L_{n}\right]\right\}
\end{aligned}
$$

where

$$
L_{n} \equiv n\left(J_{n+1 / 2}^{2}+J_{n-1 / 2} J_{n+3 / 2}\right)-\frac{2 n+3}{n+2}\left(J_{n+3 / 2}^{2}+J_{n+1 / 2} J_{n+5 / 2}\right) .
$$

For the $O$-mode, for which $e_{x}=e_{y}=0$ in this case, (12a) yields

$$
\begin{aligned}
P_{n}^{(O)}\left(N_{\|}=0\right) & =\left.\pi g_{n} \frac{x_{n}}{\left(\bar{\omega} N_{\perp}^{(O)}\right)^{2}} \frac{\partial^{2}\left|J_{n+1 / 2}\left(z_{n}\right)\right|^{2}}{\partial y_{n}^{2}}\right|_{y_{n}=0}\left|e_{z}\right|^{2} \\
& =\frac{\pi}{2} g_{n} \frac{x_{n}}{\bar{\omega}^{2}\left(N_{\perp}^{(O)}\right)^{3}}\left(J_{n+1 / 2}^{2}-J_{n-1 / 2} J_{n+3 / 2}\right),
\end{aligned}
$$

the second form of (16) following on making use of (B.12) of Appendix B and noting that $\left|e_{z}\right|^{2}=\left[N_{\perp}^{(O)}\right]^{-1}$. In $(15 \mathrm{c}, \mathrm{d})$ and (16), the argument of the Bessel functions is $x_{n}=N_{\perp}^{(i)}\left(n^{2}-\bar{\omega}^{2}\right)^{1 / 2}$, cf. $(6 b)$, and $N_{\perp}^{(i)}$ is the perpendicular (cold) refractive index of mode $i$ (Bornatici et al 1983). The result (16) for the $O$-mode has been derived earlier by solving the corresponding dispersion equation (Granata and Fidone 1991). In the limit of small finite Larmor radius effects for which a lowest-order series expansion of the Bessel functions is adequate, the absorption coefficients (11a), (15) and (16) reduce to the well-known results (Bornatici et al 1983).

\section{Asymptotic expressions for the EC absorption coefficient}

The EC absorption coefficient (11) contains an infinite sum over harmonics which, for the case of high-temperature plasmas, has to be dealt with numerically retaining a large number of terms. This complication is avoided in asymptotic expressions for the EC absorption coefficient which have been derived using the assumption that both $\bar{\omega} \gg 1$ and $\mu=m c^{2} / T_{e} \gg 1$ (Trubnikov 1979, Bornatici et al 1983 and references therein, Robinson 1985, Bertelli et al 2005) such that there is strong harmonic overlap. The asymptotic expressions of the EC absorption coefficient for propagation at an arbitrary angle to the magnetic field are listed in Bertelli et al (2005) [note that the righthand sides of Eqs.(1) and (8) of Bertelli et al (2005) must be multiplied by the factor $a(\mu) \equiv(\pi / 2 \mu)^{1 / 2} e^{-\mu} / K_{2}(\mu)$, cf. $\left.(10 b)\right]$. 
For reference, the asymptotic form of the EC absorption coefficient for perpendicular propagation is reported, i.e.,

$$
\bar{\alpha}^{(X, O)}(\bar{\omega}, \vartheta=\pi / 2)=\sqrt{\pi} \frac{a(\mu) \mu^{5 / 2}}{(2 \bar{\omega})^{2}}\left(\gamma_{0}^{2}-1\right)^{3 / 2}\left\{1, \frac{\gamma_{0}^{2}-1}{2 \bar{\omega}}\right\} e^{\Phi\left(\gamma_{0}\right)}
$$

where

$$
\begin{aligned}
\Phi\left(\gamma_{0}\right) & \equiv-\mu\left(\gamma_{0}-1\right)+2 \bar{\omega}-\bar{\omega} \gamma_{0} \ln \frac{\gamma_{0}+1}{\gamma_{0}-1} \\
& =-\mu\left(\gamma_{0}-1\right)-2 \bar{\omega} \sum_{k=2}^{\infty} \frac{1}{(2 k-1) \gamma_{0}^{2(k-1)}} .
\end{aligned}
$$

The first (second) term within the curly brackets in $(17 a)$ refers to the $X(O)$-mode and the second form of $(17 b)$ is obtained on noting that $\ln \frac{\gamma_{0}+1}{\gamma_{0}-1}=2 \sum_{k=1}^{\infty} \frac{1}{(2 k-1) \gamma_{0}^{2 k-1}}$ (Gradshteyn and Ryzhik 1994, Eq.(7.513), p.52). Upon introducing the parameter

$$
\chi \equiv \frac{9 \bar{\omega}}{2 \mu}
$$

the effective Lorentz factor $\gamma_{0}$ is solution of the equation

$$
\frac{2 \gamma_{0}}{\gamma_{0}^{2}-1}-\ln \frac{\gamma_{0}+1}{\gamma_{0}-1}=\frac{9}{2 \chi}
$$

in Trubnikov's approach (cf. Bornatici et al 1983, p. 1210), whereas

$$
\gamma_{0}\left(\equiv \gamma_{0}(\chi)\right)=\left[\frac{4}{9} \frac{\chi}{(\chi+1)^{1 / 3}}+1\right]^{1 / 2}
$$

in Robinson's treatment (Robinson 1985, Bertelli et al 2005). In particular, using (19) in $(17 a)$, yields

$$
\bar{\alpha}^{(X, O)}(\chi, \vartheta=\pi / 2)=\frac{3 \sqrt{\pi}}{2} \frac{a(\mu) \mu^{1 / 2}}{[\chi(\chi+1)]^{1 / 2}}\left\{1, \frac{1}{\mu(\chi+1)^{1 / 3}}\right\} e^{\Phi\left(\gamma_{0}(\chi)\right)} .
$$

For high frequencies and high enough electron temperatures such that $\chi \gg 1$ is also valid, in which case (19) yields

$$
\gamma_{0}(\chi) \simeq \frac{2}{3} \chi^{1 / 3}+\frac{3}{4 \chi^{1 / 3}}
$$

to lowest significant order in the factors multiplying $\exp \Phi,(20)$ reduces to

$$
\bar{\alpha}^{(X, O)}(\chi \gg 1, \vartheta=\pi / 2)=\frac{3 \sqrt{\pi}}{2} \frac{a(\mu) \mu^{1 / 2}}{\chi}\left\{1, \frac{1}{\mu \chi^{1 / 3}}\right\} e^{\Phi\left(\gamma_{0}(\chi)\right)}
$$

where now

$$
\Phi\left(\gamma_{0}(\chi)\right)=-\mu\left(\chi^{1 / 3}-1+\frac{9}{20 \chi^{1 / 3}}\right)
$$

Note that the result (22) is identical with the one obtained for $\chi \gg 1$ from Trubnikov's equation (18) (Bornatici et al 1983, Eq.(3.2.20), p.1210) which in this limit yields

$$
\gamma_{0}(\chi) \simeq \frac{2}{3} \chi^{1 / 3}+\frac{3}{5 \chi^{1 / 3}}
$$

as the term of order $\chi^{-1 / 3}$ in the expressions for $\gamma_{0}(\chi)$ does not contribute to $\Phi$ in that order. 


\section{Numerical analysis}

The accuracy of the quasi-exact treatment of the EC absorption, cf. Eqs.(11)-(12), (15)-(16), has been assessed numerically and the results of this analysis are presented and discussed in the following. The parameters considered have been chosen to be representative for those of interest for hot fusion plasmas, viz. temperatures in the range of 30 to $50 \mathrm{keV}$ (effectively, temperatures between 10 and $100 \mathrm{keV}$ have been explored) and modestly high frequency, $\bar{\omega}=5$ being used here as a typical example. In addition, results obtained from the asymptotic formulae (see Section 3) are given, for comparison, although it must be emphasized that the underlying approximations, at best, are marginally valid in the parameter range under consideration.

Let us start with the case of perpendicular propagation for which the quasi-exact (QE) absorption coefficient for the $X$-mode (cf. Eqs.(11a),(15) and (16)) is shown in Fig.1a as a function of electron temperature $T_{e}$ for $\bar{\omega}=5$; the results obtained from the asymptotic expressions by Robinson (1985), cf. Eqs.(17b), (19) and (20), and by Trubnikov (1979), cf. Eqs.(17) and (18) (Bornatici et al 1983), as well as those corresponding to the limit $\chi \gg 1$ of both those approximations, cf. Eqs.(22), and the exact result, cf. Eqs.(11) along with (9), see also Bornatici and Ruffina (1989) are also given. The relative error of the different approximations with respect to the exact value of the absorption coefficient is shown in Fig.1b. The analogous numerical analysis for the $O$-mode is given by Figs.2. In particular, from Figs.1b and $2 \mathrm{~b}$ it appears that (i) the QE result is quite accurate for both modes, underestimating the exact result by less than 1\%; (ii) for $T_{e}$ between 25 and $90 \mathrm{keV}$ Robinson's asympotic result (20) underestimates the exact value of the absorption coefficient of the $X$-mode by less than $10 \%$, whereas for the $O$-mode, it overestimates the exact value by less than about $20 \%$ for $T_{e}$ between 15 and (more than) $100 \mathrm{keV}$; (iii) Trubnikov's asymptotic result (17) along with (18) is more (less) accurate than the Robinson's for the $O$-mode ( $X$-mode); (iv) both Trubnikov's and Robinson's asymptotic treatment tend to overestimate the absorption by more than $25 \%$ for temperature $T_{e} \lesssim 15 \mathrm{keV}$, i.e., for temperatures for which the absorption is weak and harmonic overlap tends to be reduced, cf. Figs.1a and 2a; (v) for the $X$-mode, the results obtained adopting Eqs.(22) as valid in the limit $\chi \gg 1$ are accurate to within about $10 \%$ for $T_{e} \gtrsim 60 \mathrm{keV}$ and tend to be more accurate than Trubnikov's result for $T_{e} \gtrsim 50 \mathrm{keV}$ and Robinson's result for $T_{e} \gtrsim 80 \mathrm{keV}$, cf. Fig.1b; for the $O$-mode, instead, Eqs.(22) are less accurate than Robinson's and Trubnikov's result for $T_{e} \gtrsim 50 \mathrm{keV}$, cf. Fig.2b. It is to be noted that the differences between the results given by the asymptotic formulae, are mainly due to using different values of $\gamma_{0}$ in the factor $\gamma_{0}^{2}-1$ of Eq. $(17 a)$.

As for the propagation at an arbitrary angle with respect to the magnetic field, cf. Eqs.(11)-(12), the QE result for the angular distribution of the absorption at $\bar{\omega}=5$ is shown in Fig.3a for the $X$-mode, for electron temperatures $T_{e}=30 \mathrm{keV}$ and $40 \mathrm{keV}$, along with the corresponding results obtained from the asymptotic formulae by Robinson (1985) and by Trubnikov (1979); see also Bertelli et al (2005). The 
curves labelled "QE" refer to the absorption coefficient $2 \operatorname{Imk}$ (normalized to $\omega_{p}^{2} / c \omega_{c}$ ) as obtained from the numerical solution of the (quasi-exact) dispersion relation (Granata and Fidone 1991, Albajar 2001). The relative deviation of both the QE result and the asymptotic results with respect to the "QE" result is shown in Fig.3b. The analogous numerical analysis for the $O$-mode is given in Figs.4. In particular, for the $X$-mode, from Fig.3b it appears that (i) the QE analytical absorption coefficient obtained from the electromagnetic energy balance is practically identical to the one obtained by solving numerically the dispersion relation; (ii) for almost perpendicular propagation, i.e., for the angular range $75^{\circ} \lesssim \vartheta \leq 90^{\circ}$ where absorption is strongest, the accuracy of Robinson's asymptotic result is better than $10 \%$ for $T_{e} \approx 30$ to $40 \mathrm{keV}$ and better than Trubnikov's approximation for $\vartheta \gtrsim 83^{\circ}$ (see also Fig.1b); (iii) over most of the angular range, namely, for $10^{\circ} \lesssim \vartheta \lesssim 75^{\circ}$, Trubnikov's asymptotic result is better than Robinson's, the accuracy of the former being better than $10 \%$, whereas Robinson's result becomes quite inaccurate specifically for propagation at small angles, typically, for $\vartheta \lesssim 20^{\circ}$. Turning now to the $O$-mode, from Fig.4a it appears that the characteristic nonmonotonous angular distribution of the $O$-mode absorption is accounted for by both the QE and "QE" evaluation, as well as by Robinson's asymptotic treatment. Trubnikov's asymptotic result, instead, underestimates the $O$-mode absorption significantly, with the only exception of perpendicular propagation, cf. Fig.2b, and fails to reproduce the nonmonotonous behaviour of the angular distribution (Bertelli et al 2005). As it appears from Fig.4b, (i) the QE absorption coefficient again agrees very well with the "QE" value obtained from solving the (quasi-exact) dispersion relation numerically (Granata and Fidone 1991, Albajar 2001); (ii) Robinson's asymptotic evaluation is accurate by better than about $20 \%$ over most of the angular range, with the exception of the propagation at small angles $\left(\vartheta \lesssim 25^{\circ}\right)$, for which it overestimates the $O$-mode absorption by more than $30 \%$.

\section{Concluding remarks}

In conclusion, the quasi-exact (QE) analytical result presented here provides an excellent approximation to the EC absorption coefficient of a Maxwellian plasma. Notwithstanding the lower accuracy of both Robinson's and Trubnikov's asymptotic results compared to the QE treatment, the noticeable practical advantage of these asymptotic expressions for the absorption coefficient is that they are free from the sum over harmonics (Bertelli et al 2005), as present in the QE absorption coefficient. Since, overall, Robinson's asymptotic form is a quite reasonable approximation to the EC absorption coefficient in most of the relevant parameter range (effectively, a better one than Trubnikov's form), this form can be expected to be a useful starting point for calculating the effective EC wave power loss from a large hot plasma (cf. Albajar et al 2002). This fact has been confirmed by solving the radiative transfer equation for fusion plasma parameters, showing in particular that using this approach calculation times are reduced typically by two orders of magnitude with respect to using the QE form (and 
to the "QE" approach) (Albajar et al 2006).

\section{Figure captions}

Figure 1a. The (normalized) quasi-exact (QE) absorption coefficient of the $X$-mode, for perpendicular propagation, Eqs.(11),(15) and (16), as a function of the electron temperature $T_{e}$, for $\left(\omega_{p} / \omega_{c}\right)^{2}=0.1$ and $\bar{\omega}\left(\equiv \omega / \omega_{c}\right)=5$. Also shown are the exact result (Eqs.(11) along with (9), E-curves) and the asymptotic results of both Robinson (Eq.(20), R-curve) and Trubnikov (Eqs.(17) and (18), T-curve), as well as the $\chi \gg 1$ result, Eq.(22).

Figure 1b. Relative error $\Delta \equiv\left(\alpha_{A}-\alpha_{E}\right) / \alpha_{E}$ of the absorption coefficient of the $X$-mode (the subscripts $\mathrm{A}(=\mathrm{QE}, \mathrm{R}, \mathrm{T}$ and $\chi \gg 1)$ and $\mathrm{E}$ are the same as the labels in Fig.1a).

Figure 2a. The same as Fig.1a for the $O$-mode.

Figure 2b. The same as Fig.1b for the $O$-mode.

Figure 3a. Quasi-exact (QE) absorption coefficient of the $X$-mode as a function of the angle of propagation with respect to the magnetic field, for $\left(\omega_{p} / \omega_{c}\right)^{2}=0.1$, $\bar{\omega}\left(\equiv \omega / \omega_{c}\right)=5$ and electron temperatures $T_{e}=30 \mathrm{keV}$ (dashed curve) and $40 \mathrm{keV}$ (full curve). Also shown is the absorption coefficient $2 \operatorname{Imk}$ (normalized to $\omega_{p}^{2} / c \omega_{c}$ ), "QE"-curve, as given by the numerical solution of the (quasi-exact) dispersion relation (Granata and Fidone 1991, Albajar 2001), as well as the asymptotic results of both Robinson (R-curve) and Trubnikov (T-curve) (Bertelli et al 2005).

Figure 3b. Relative deviation of both the QE results and Robinson's and Trubnikov's asymptotic results for the absorption coefficient of the $X$-mode with respect to the "QE" result, for the same parameters as Fig.3a .

Figure 4a. The same as Fig.3a for the $O$-mode.

Figure $4 \mathbf{b}$. The same as Fig.3b for the $O$-mode (Trubnikov's asymptotic result being omitted). 

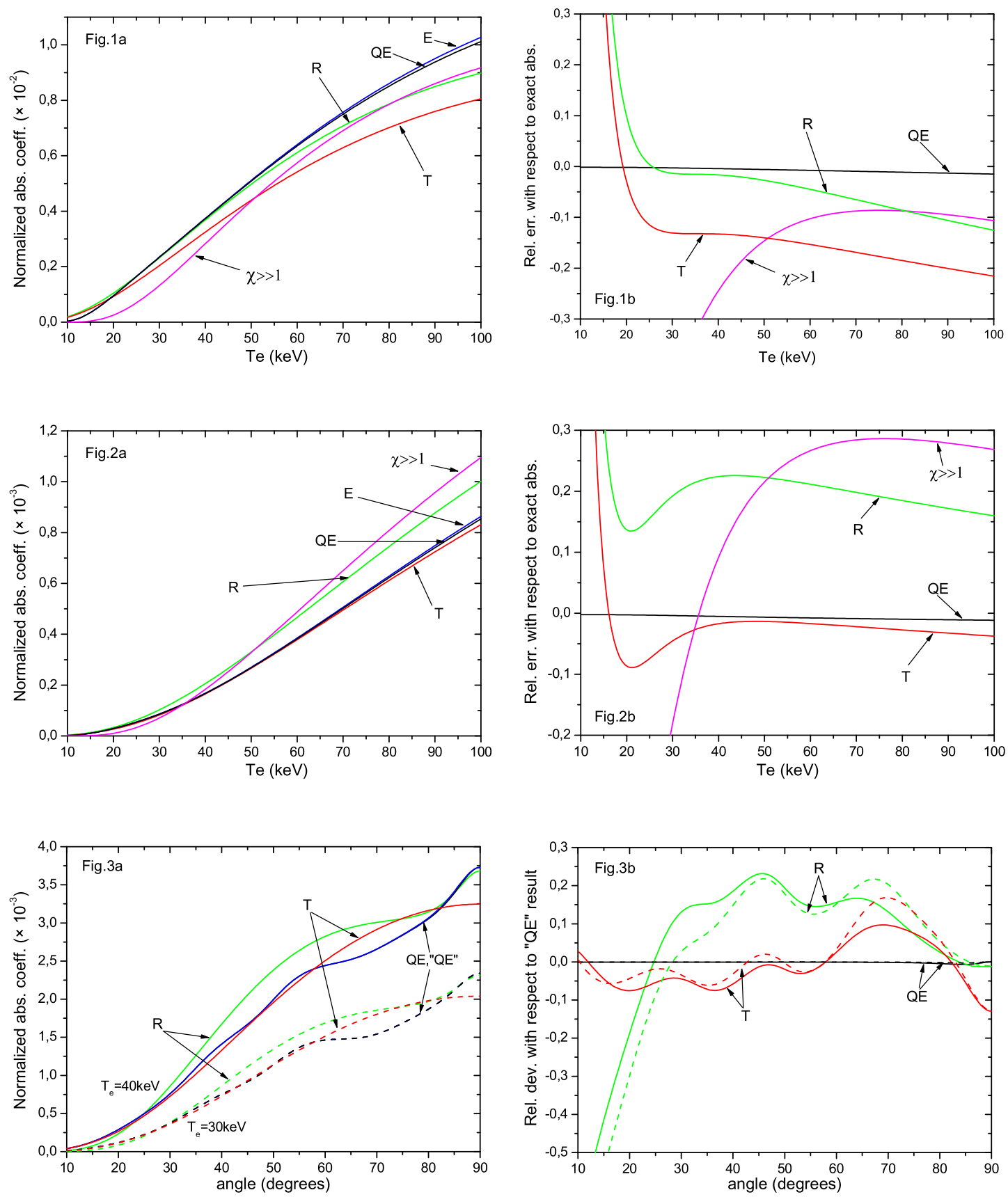

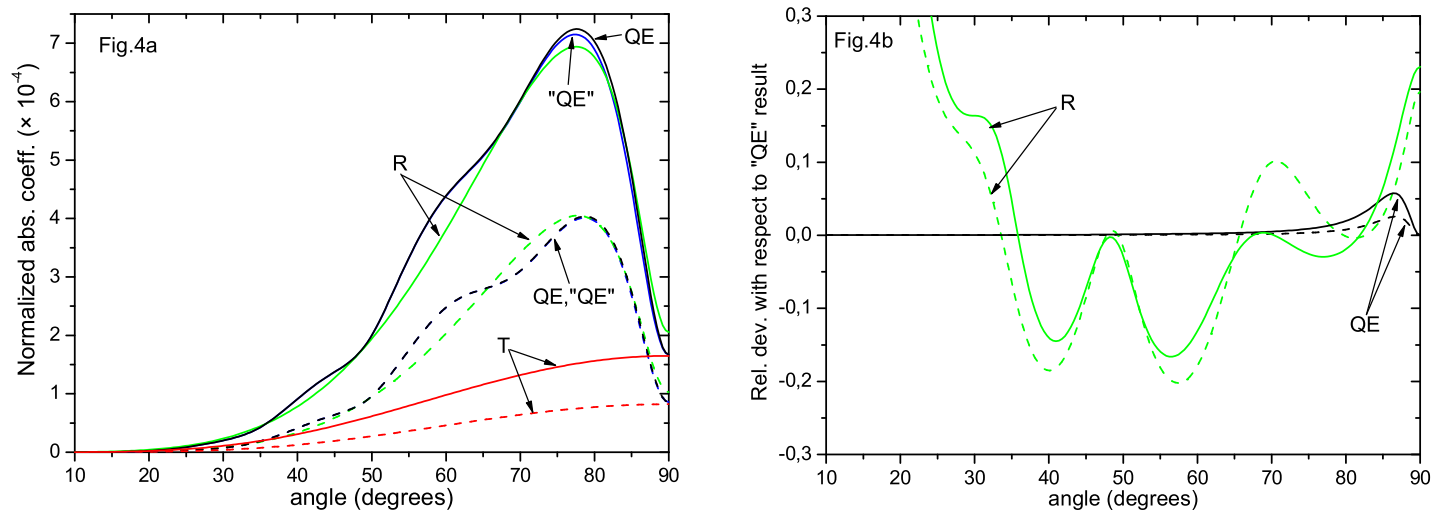

\section{Appendix A. Bateman's expansion of Bessel functions}

Here we collect the two forms of Bateman's expansion of Bessel functions which we have used in the evaluation of the integral (11b). Bateman's expansion (cf. Watson (1944), Eq.(1) on p. 370) concerns the product of two Bessel functions of argument $x$ and (non-negative integer) order $\mu$ and $\nu$ which typically is expressed in terms of a series of Bessel functions of argument $2 x$ and order $\mu+\nu+1+2 m$, the sum being over $m=0,1,2, \ldots$

For the specific case of the square of a Bessel function of (non-negative) integer order one has (Granata 1990)

$$
\begin{aligned}
x J_{n}^{2}(x) & =\sum_{m=0}^{\infty} g_{n, m} J_{2 n+4 m+1}(2 x), \quad g_{n, m}=\frac{(2 n+4 m+1)(2 n+2 m) !(2 m) !}{\left[2^{n+2 m}(n+m) !(m) !\right]^{2}} \\
& =g_{n}\left[J_{2 n+1}(2 x)+\frac{2 n+5}{4(n+1)} J_{2 n+5}(2 x)+\ldots\right], \quad g_{n}\left(\equiv g_{n, 0}\right)=\frac{(2 n+1) !}{\left(2^{n} n !\right)^{2}}
\end{aligned}
$$

to be referred to as the first form of Bateman's expansion. One should note that here the general term of the series contains a Bessel function the (odd) integer order of which is even higher by 4 than the order of the Bessel function in the preceding term which guarantees a rapid convergence of the series (A.1). More quantitatively, retaining the lowest order term of the expansion is accurate to better than $1 \%$ for $x \lesssim 6$ and $n \geq 4$ as one can see from Fig.(A1) where the ratio $r_{n}(x) \equiv J_{2 n+5}(x) / J_{2 n+1}(x)$ of the Bessel functions present in the first two terms of the series is shown. In particular, if finite Larmor radius (FLR) effects are small, i.e., for $x \ll 1$, one has $r_{n}(x) \propto x^{4}$, that is, FLR effects are fully accounted for to first and second order by just retaining the lowest order term in the expansion (A.1).

For the product of two Bessel functions of (positive) integer order different by 2 , one has (Granata 1990)

$$
\begin{aligned}
x J_{n-1}(x) J_{n+1}(x) & =\frac{g_{n}}{n+1}\left\{n J_{2 n+1}(2 x)\left[1+\frac{n(2 n+5)}{4(n+2)(n+3)} r_{n}(2 x)\right]+\right. \\
& \left.-\frac{2 n+3}{n+2} J_{2 n+3}(2 x)\left[1+\frac{3(n+2)(2 n+7)}{4(n+3)(n+4)} r_{n+1}(2 x)\right]+\ldots\right\}
\end{aligned}
$$




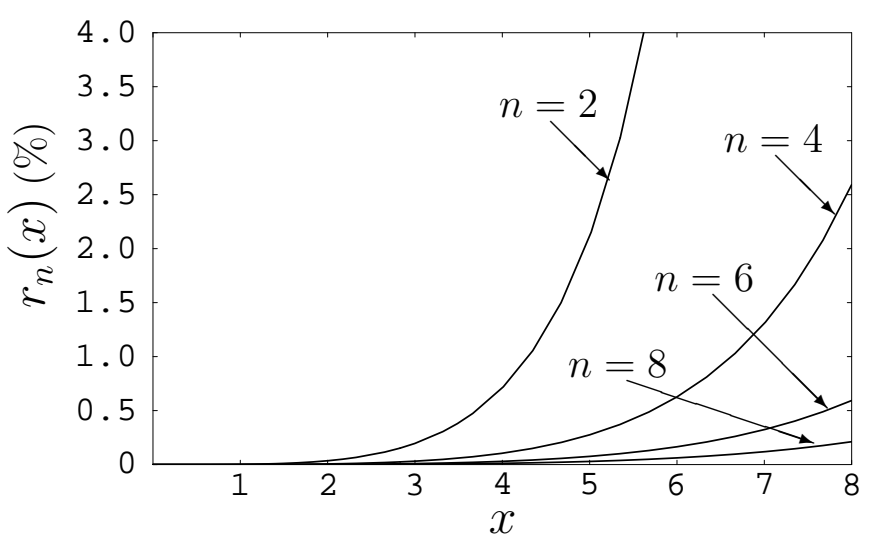

Figure A1. The ratio $r_{n}(x) \equiv J_{2 n+5}(x) / J_{2 n+1}(x)$ as a function of $x$ for different values of $n$.

(second form of Bateman's expansion). It appears that in this case the order of the Bessel functions in the expansion progresses by 2 and, if the two lowest-order terms containing the Bessel functions of order $(2 n+1)$ and $(2 n+3)$ are retained in (A.2), the accuracy of this approximation is again given by the quantity $r_{n}(x)$.

\section{Appendix B. The evaluation of the integral (11b)}

The integral (11b) comprises two terms related to the even (in $t$ ) and odd (in $t$ ) parts of the polarization factor $(8 a)$,

$$
\begin{aligned}
P_{n} & =2 \int_{0}^{1} d t\left[\left|\mathbf{e} \cdot \mathbf{V}_{n}^{*}\right|_{\text {even }}^{2} \cosh \left(y_{n}\left(N_{\|}\right) t\right)-\left|\mathbf{e} \cdot \mathbf{V}_{n}^{*}\right|_{\text {odd }}^{2} \sinh \left(y_{n}\left(N_{\|}\right) t\right)\right] \\
& =2 \int_{0}^{1} d t\left[\left|\mathbf{e} \cdot \mathbf{V}_{n}^{*}\right|_{\text {even }}^{2}-\left|\mathbf{e} \cdot \mathbf{V}_{n}^{*}\right|_{\text {odd }}^{2} \frac{1}{t} \frac{\partial}{\partial y_{n}\left(N_{\|}\right)}\right] \cosh \left(y_{n}\left(N_{\|}\right) t\right) .
\end{aligned}
$$

Hence, using (8a) explicitly, the calculation of the expression (B.1) amounts to the evaluation of the integrals

$$
\left(\begin{array}{c}
i_{1} \\
i_{2} \\
i_{3}
\end{array}\right) \equiv \int_{0}^{1} d t\left(\begin{array}{c}
{\left[J_{n}\left(x \sqrt{1-t^{2}}\right)\right]^{2}} \\
\sqrt{1-t^{2}}\left[x \sqrt{1-t^{2}} J_{n-1}\left(x \sqrt{1-t^{2}}\right) J_{n+1}\left(x \sqrt{1-t^{2}}\right)\right] \\
{\left[t J_{n}\left(x \sqrt{1-t^{2}}\right)\right]^{2}}
\end{array}\right) \cosh (y t),(
$$

related to the even (in $t)$ part of $(8 a)$, and

$$
i_{4} \equiv \int_{0}^{1} d t t\left[J_{n}\left(x \sqrt{1-t^{2}}\right)\right]^{2} \sinh (y t)
$$

for the odd part of $(8 a)$, with $i_{p} \equiv i_{p}(x, y)$ and $p=1,2,3,4$. Both $i_{3}$ and $i_{4}$ can be expressed in terms of $i_{1}$, i.e.,

$$
i_{3}=\frac{\partial^{2} i_{1}}{\partial y^{2}}, \quad i_{4}=\frac{\partial i_{1}}{\partial y},
$$


while the evaluation of both $i_{1}$ and $i_{2}$ can be carried out on making use of Bateman's expansion of the product of two Bessel functions. With the first form of Bateman's expansion, to lowest order, i.e. with

$$
x J_{n}^{2}(x)=g_{n} J_{2 n+1}(2 x), \quad g_{n} \equiv \frac{(2 n+1) !}{\left(2^{n} n !\right)^{2}},
$$

cf. (A.1) of the Appendix A, the integral $i_{1}$ of (B.2) can be conveniently written

$$
\begin{aligned}
i_{1}(x, y)=\frac{g_{n}}{x} \int_{0}^{1} \frac{d t}{\sqrt{1-t^{2}}} J_{2 n+1}\left(2 x \sqrt{1-t^{2}}\right) \cosh (y t) \\
=\frac{\pi}{2} \frac{g_{n}}{x} \times\left\{\begin{array}{l}
\left|J_{n+1 / 2}(z)\right|^{2}, \quad z \equiv \frac{1}{2}\left(\sqrt{4 x^{2}-y^{2}}+i y\right), \\
\text { for } 4 x^{2}-y^{2}>0, \text { i.e., }(c f .(6 b) \text { and }(11 c)), 2 n_{0}>\mu N_{\|} / N_{\perp} ; \\
I_{n+1 / 2}\left(z^{+}\right) I_{n+1 / 2}\left(z^{-}\right), \quad z^{ \pm} \equiv \frac{1}{2}\left(y \pm \sqrt{y^{2}-4 x^{2}}\right), \\
\text { for } y^{2}-4 x^{2}>0, \text { i.e., } 2 n_{0}<\mu N_{\|} / N_{\perp} .
\end{array}\right.
\end{aligned}
$$

The inequalities occurring in (B.6), respectively, characterize propagation at large (upper entry) and small (lower entry) angles to the magnetic field.

The result of the upper entry of (B.6) is obtained on applying Eq.(6.739) of Gradshteyn and Ryzhik (1994), along with the property that $J_{\nu}\left(z^{*}\right)=\left[J_{\nu}(z)\right]^{*}$, cf. Eq.(9.1.40), p. 361 of Abramowitz and Stegun (1970), the argument of the Bessel function being complex. The result of the lower entry of (B.6), on the other hand, follows from (B.5) with $z \rightarrow i z^{+}$and $z^{*} \rightarrow-i z^{-}=(i z)^{*}$, so that $J_{n+1 / 2}(z) J_{n+1 / 2}\left(z^{*}\right) \rightarrow$ $J_{n+1 / 2}\left(i z^{+}\right)\left[J_{n+1 / 2}\left(i z^{-}\right)\right]^{*}=I_{n+1 / 2}\left(z^{+}\right) I_{n+1 / 2}\left(z^{-}\right)$, the equality following on expressing the Bessel function of imaginary argument in term of the modified Bessel function of the first kind of real argument, i.e., $J_{n+1 / 2}(i z)=e^{i\left(n+\frac{1}{2}\right) \frac{\pi}{2}} I_{n+1 / 2}(z)$, cf. Eq.(9.6.3), p. 375 of Abramowitz and Stegun (1970). It is worth noting that, whereas Bateman's expansion is such that a bilinear form of Bessel functions is reduced to a linear form, the integration (B.5) again produces a bilinear form of Bessel functions.

As for the integral $i_{2}$ in (B.2), on making use of the second form of Bateman's expansion to second order, cf. Eq.(A.2) of the Appendix A, i.e.,

$$
x J_{n-1}(x) J_{n+1}(x)=\frac{g_{n}}{n+1}\left[n J_{2 n+1}(2 x)-\frac{2 n+3}{n+2} J_{2 n+3}(2 x)\right],
$$

one has

$$
i_{2}=\frac{g_{n}}{n+1}\left[n i_{2}^{(1)}-\frac{2 n+3}{n+2} i_{2}^{(2)}\right]
$$

where

$$
\left(\begin{array}{c}
i_{2}^{(1)} \\
i_{2}^{(2)}
\end{array}\right) \equiv \int_{0}^{1} d t \sqrt{1-t^{2}}\left(\begin{array}{c}
J_{2 n+1}\left(2 x \sqrt{1-t^{2}}\right) \\
J_{2 n+3}\left(2 x \sqrt{1-t^{2}}\right)
\end{array}\right) \cosh (y t)
$$




$$
=\left(1-\frac{\partial^{2}}{\partial y^{2}}\right) \int_{0}^{1} d t \frac{1}{\sqrt{1-t^{2}}}\left(\begin{array}{c}
J_{2 n+1}\left(2 x \sqrt{1-t^{2}}\right) \\
J_{2 n+3}\left(2 x \sqrt{1-t^{2}}\right)
\end{array}\right) \cosh (y t) .
$$

The two integrals (B.8) are of the same type as the integral occurring in (B.5); thus

$$
i_{2}^{(1)}=\frac{x}{g_{n}}\left(1-\frac{\partial^{2}}{\partial y^{2}}\right) i_{1}(x, y)
$$

and

$$
i_{2}^{(2)}=\left(1-\frac{\partial^{2}}{\partial y^{2}}\right)\left(\frac{x}{g_{n}} i_{1}(x, y)\right)_{n \rightarrow n+1}=\frac{\pi}{2}\left(1-\frac{\partial^{2}}{\partial y^{2}}\right) \times\left\{\begin{array}{l}
\left|J_{n+3 / 2}(z)\right|^{2} \\
I_{n+3 / 2}\left(z^{+}\right) I_{n+3 / 2}\left(z^{-}\right)
\end{array}\right.
$$

the upper and lower entry of (B.10) corresponding, respectively, to the conditions indicated in (B.6). With (B.9) and (B.10), (B.7) yields

$$
i_{2}=\frac{\pi}{2} \frac{g_{n}}{n+1}\left(1-\frac{\partial^{2}}{\partial y^{2}}\right) \times\left\{\begin{array}{l}
{\left[n\left|J_{n+1 / 2}(z)\right|^{2}-\frac{2 n+3}{n+2}\left|J_{n+3 / 2}(z)\right|^{2}\right]} \\
\text { for upper entry conditions of }(\mathrm{B} .6) ; \\
{\left[n I_{n+1 / 2}\left(z^{+}\right) I_{n+1 / 2}\left(z^{-}\right)-\frac{2 n+3}{n+2} I_{n+3 / 2}\left(z^{+}\right) I_{n+3 / 2}\left(z^{-}\right)\right]} \\
\text {for lower entry conditions of (B.6). }
\end{array}\right.
$$

For the case of perpendicular $\left(N_{\|}=0\right)$ propagation, for which $y_{n}=0$, cf. $(11 c)$, the second derivatives with respect to $y$ occurring in both $i_{3}$, cf. (B.4), and $i_{2}$, cf. (B.11), are to be evaluated for $y=0$, with the result that

$$
\left.\frac{\partial^{2}}{\partial y^{2}}\left|J_{n+1 / 2}(z)\right|^{2}\right|_{y=0}=\frac{1}{2}\left[J_{n+1 / 2}^{2}(x)-J_{n-1 / 2}(x) J_{n+3 / 2}(x)\right]
$$

and

$$
\left.\frac{\partial^{2}}{\partial y^{2}}\left|J_{n+3 / 2}(z)\right|^{2}\right|_{y=0}=\frac{1}{2}\left[J_{n+1 / 2}^{2}(x)+J_{n+3 / 2}^{2}(x)-\frac{2 n+3}{x} J_{n+1 / 2}(x) J_{n+3 / 2}(x)\right],
$$

(B.13) following from (B.12) with $n \rightarrow n+1$ along with the recurrence relation $J_{n+1}(x)=\frac{2 n}{x} J_{n}(x)-J_{n-1}(x)$. Note also that $i_{4}$, defined in (B.3), is identically zero for $y=0$.

\section{References}

Abramowitz M and Stegun I A 1970 Handbook of Mathematical Functions (Dover Publi., New York) Albajar F 2001 Radiation Transport Modelling in a Tokamak Plasma: Application to Performance Prediction and Design of Future Machines, Ph.D. Thesis, URN http://www.tdx.cesca.es/TDX0114104-103202/ , ISBN B. 10751-2004/84-688-5356-9.

Albajar F, Bornatici M and Engelmann F 2002, Nucl. Fusion 42670

Albajar F et al 2006, in preparation

Bertelli N, Bornatici M and Engelmann F 2005 Phys. Letters A 347 114; and also Erratum 2006 Phys. Letters A 350431 
Bornatici M, Cano R, De Barbieri O and Engelmann F 1983 Nucl. Fusion 231153 and references therein

Bornatici M and Engelmann F 1994 Phys. Plasmas 1189

Bornatici M and Ruffina U 1989 Phys. Fluids B1 242

Gradshteyn I S and Ryzhik I M 1994 Table of Integrals, Series and Products (Academic Press) p 777

Granata G 1990 Report EUR-CEA-FC-1401

Granata G and Fidone I 1991 J. Plasma Phys. 45361

Robinson P A 1985 Plasma Phys. Contr. Fusion 271037

Trubnikov B A 1979 Review of Plasma Physics (M. A. Leontovich Ed.), vol 7, Consultants Bureau, New York 345

Watson G N 1994 A Treatise on the Theory of Bessel Functions (Cambridge Univ. Press) p 370 